\title{
A single explosion model for the supernova remnant 3C 400.2
}

\author{
P. F. Velázquez ${ }^{1, \star}$, E. de la Fuente ${ }^{1, \star \star}$, M. Rosado ${ }^{1}$, and A. C. Raga ${ }^{2}$ \\ 1 Instituto de Astronomía - Universidad Nacional Autónoma de México, Apdo. Postal 70-264, \\ Ciudad Universitaria, C.P. 04510, México D.F., México \\ e-mail: pablov, edfuente, margarit, raga@astroscu.unam.mx \\ 2 Instituto de Ciencias Nucleares -Universidad Nacional Autónoma de México, Apdo. Postal 70-543, \\ Ciudad Universitaria, C.P. 04510, México D.F., México
}

Received 10 April 2001 / Accepted 31 July 2001

\begin{abstract}
We present a numerical simulation carried out in order to explain the complex morphology of the supernova remnant (SNR) 3C 400.2. Numerical simulations were done employing the new yguazú-a code (Raga et al. 2000). In the radio continuum, this remnant seems to be formed by two shells, which are partially overlapping (Dubner et al. 1994). Taking into account observational evidence such as a previous HI study (Giacani et al. 1998), we find that an adequate model for describing the morphology of the SNR 3C 400.2 is obtained by assuming that a single supernova ( $\mathrm{SN}$ ) explosion took place in a dense medium and close to an interface separating this medium from a lower density region. Besides, we also present a new deeper CCD H $\alpha$ image mosaic of the north-west region of this SNR, which is composed of three single images. From the numerical results, we generated $\mathrm{H} \alpha$ emission maps, obtaining a good agreement with the observations.
\end{abstract}

Key words. ISM: supernova remnants - supernovae individual 3C 400.2 - hydrodynamics methods: numerical - methods: observational

\section{Introduction}

SNRs expanding into an isotropic and homogeneous medium have spherical shapes. However, the interaction of a SNR with the circumstellar medium, which could have been modified by the SN progenitor, or the presence of a compact object (formed after the SN explosion) which injects energy and particles into the remnant, can produce morphologies which are far from spherical, such as the case of the SNR W50 (Dubner et al. 1998; Velázquez \& Raga 2000).

3C 400.2 (G53.6-2.2, SNR 053.6-02.2) is an extended Galactic non-thermal radio source (Milne 1970) closely centered on J2000: $19^{\mathrm{h}} 38^{\mathrm{m}} 7^{\mathrm{s}}, 17^{\circ} 15^{\prime}$, which was first identified as a SNR by Holden \& Caswell (1969). This SNR shows a typical non-thermal radio spectral index in the $0.6 \leq \alpha \leq 0.76$ range $\left(\mathrm{S}_{\nu} \propto \nu^{-\alpha}\right.$, e.g. Milne 1970; Goss et al. 1975; Dubner et al. 1994). This remnant belongs to the group of SNRs which are characterized by a synchrotron radio shell and thermal X-ray emission, filling up their interiors.

Radio continuum studies at 318 and $430 \mathrm{MHz}$ (Dickel \& De Noyer 1975), $610 \mathrm{MHz}$ (Goss et al. 1975), 1.7 and

\footnotetext{
Send offprint requests to: P. F. Velázquez, e-mail: pablov@astroscu. unam.mx

* Postdoctoral Fellow of CONICET, Argentina.

** Also, Facultad de Ciencias - Universidad Nacional Autónoma de México.
}

2.7 GHz (Willis 1973) and $4.85 \mathrm{GHz}$ (Condon et al. 1989) have shown that the radio morphology of $3 \mathrm{C} 400.2$ is a well-developed shell of $30^{\prime}$ diameter, having an irregular structure which is brightest in the north-east and north-west regions.

From high-resolution radio observations, carried out by Dubner et al. (1994), the complex morphology of this SNR can be described as two partially overlapping nonthermal shells: a large shell located in the southeast region with an angular size of $44^{\prime}$, centered near $19^{\mathrm{h}} 38^{\mathrm{m}} 53^{\mathrm{s}}$, $17^{\circ} 12^{\prime} 55^{\prime \prime}$; and a small one in the north-west region with an angular diameter of $28^{\prime}$, centered near $19^{\mathrm{h}} 38^{\mathrm{m}} 10^{\mathrm{s}}$, $17^{\circ} 17^{\prime} 52^{\prime \prime}$. The two shells overlap each other in the northwest of 3C 400.2. In their analysis, Dubner et al. (1994) explore different possible scenarios to explain the strange morphology of 3C 400.2, suggesting that this object could be the result of the interaction between two SNR, or that it could be the consequence of a single SN explosion occurring into a dense cloud. They also suggest that 3C 400.2 could be the result of a coincidence on the sky of two different SNRs.

There are several theoretical models describing the interaction between two SNRs or a SNR expanding in stratified medium (Ikeuchi 1978; Jones et al. 1979; TenorioTagle et al. 1985 and Arthur \& Falle 1991), but they do not apply these results to the case of SNR 3C 400.2. 
By means of an analysis of the HI distribution around this remnant, Giacani et al. (1998) find that the double shell structure could be produced by a single SN explosion, initially expanding into a dense medium $\left(n \simeq 20 \mathrm{~cm}^{-3}\right)$. Then, in its expansion, the SNR shock wave encounters a region with low density, producing a blow out, which breaks the SNR shell generating a bubble and giving 3C 400.2 the appearance of two overlapping SNR shells.

Kinematical studies presented by Rosado (1983) and spectroscopical studies carried out by Sabbadin \& D'Odorico (1976), Blair \& Long (1988) and Long \& Blair (1991) show that 3C 400.2 is an old SNR (approximately $1 \times 10^{5}$ yr, e.g. Rosado 1983), probably in the radiative phase of SNR evolution (Woltjer 1970, 1972).

The optical emission of 3C 400.2 was first reported by van den Bergh et al. (1973). The South West region was revealed by Rosado (1983), who presented $\mathrm{H} \alpha$, [SII] and [OIII] plates, and by Blair \& Long (1988), who showed a $\mathrm{H} \alpha+[\mathrm{NII}]$ image. Winkler et al. (1993) presented the first CCD mosaic covering the full extent of 3C 400.2 in the continuum $(6100 \AA), \mathrm{H} \alpha+[\mathrm{NII}] 6583 \AA$, [SII] $6717 \AA$, and [OIII] $5007 \AA$. The optical emission has a partial ring structure, located in the region to the northwest of the radio shell.

The X-ray emission has a probable thermal origin and fills up the interior of this SNR, its peak flux coinciding with the region where the two apparent radio shells partially overlap (Matsui \& Long 1985; Seward 1990; Long et al. 1991). This emission was interpreted in terms of the evaporation of small clouds which are embedded in the surrounding ISM, by White \& Long (1991) (also Long et al. 1991). However, in their model, they employ densities which are two orders of magnitude lower than the densities calculated by Giacani et al. (1998). Another possible model for explaining the X-ray emission of 3C 400.2 is the one of Shelton et al. (1999), in which thermal conduction was considered as a mechanism to transport energy outwards and avoid a strong reduction of the central SNR density (see also Cox et al. 1999). They applied this model to the case of the SNR W44.

Our aim is to analyze if a single $\mathrm{SN}$ explosion scenario can explain the characteristics of the SNR 3C 400.2. To this effect, we have carried out numerical simulations using the new yguazú code (Raga et al. 2000) for describing the evolution and morphology of this remnant. Due to the fact that this SNR seems to be expanding in a dense medium, the code includes radiative losses. We also simulate the $\mathrm{H} \alpha$ emission in order to compare the numerical results with our new north-west H $\alpha$ CCD mosaic and with other observations in the literature.

The work is organized as follows. In Sect. 2 we describe the observations and the data-reduction process. In Sect. 3, we list the assumptions and initial conditions of the numerical simulation. In Sect. 4, we show the results of both the simulated and observed $\mathrm{H} \alpha$ maps. Finally, the conclusions are given in Sect. 5 .

\section{Observations}

3C 400.2 was observed in 1998 June 24, with the UNAM Scanning Fabry-Perot Interferometer PUMA (Rosado et al. 1995) at the f/7.5 Cassegrain focus of the $2.1 \mathrm{~m}$ telescope of the Observatorio Astronómico Nacional, located in the San Pedro Mártir Mountains, Baja California, México (OAN-SPM). A liquid nitrogen cooled thinned Tektronix CCD detector with $1024 \times 1024$ pixels was employed. In order to increase the signal/noise ratio of the observations, the CCD format has been binned by four, resulting in images of $256 \times 256$ pixels with an angular resolution of $2.36^{\prime \prime}$ pix $^{-1}$ and covering a field of $10^{\prime}$ diameter. The PUMA equipment was used in its "direct image" mode, i.e., placing the Fabry-Perot interferometer outside the optical path of the rays and consequently, using the PUMA only as a focal reducer. The interference filter used in the observations is centered on $\mathrm{H} \alpha(\lambda 6563 \AA)$ and has a bandpass of $20 \AA$. With this instrumental set-up we obtained images at three different positions of the northwest region of the SNR 3C 400.2. Each image has an exposure time of 60 seconds. The observations in PUMA's "interferometric" mode, i.e., with the optical path of the rays passing through the Fabry-Perot interferometer, will be presented in a future paper. The data reduction was carried out using the specific reduction package CIGALE (Le Coarer et al. 1993) and some IRAF ${ }^{1}$ routines.

\section{Numerical model}

\subsection{The yguazú-a code}

For the numerical simulations described in this paper, we have used the recently developed yguazú- $a$ code. This code solves the gas dynamic equations together with additional rate equations describing the relevant microphysical processes. The gas dynamic equations are integrated with a second order accurate (in space and time) implementation of the "flux-vector splitting" algorithm of Van Leer (1982), and the rate equations are integrated with an unconditionally stable, semi-implicit method (described in detail by Raga et al. 2000). The code uses a binary, hierarchical adaptive grid, and both 2D (plane or axisymmetric) and $3 \mathrm{D}$ versions of the code are available (for a detailed description of the adaptive grid, see Raga et al. 2000).

For our supernova remnant simulations, we have used a version of the yguazú-a code which integrates the gas dynamic equations together with one rate equation following the fraction of neutral hydrogen. The parametrized radiative cooling function of Raga et al. (1999), which is computed as a function of the density, temperature and neutral fraction has been included in the energy equation. This version of the yguazú- $a$ code has already been used for simulations of jet flows by Raga et al. (2001).

\footnotetext{
${ }^{1}$ IRAF is distributed by the National Optical Astronomy Observatories, operated by the Association of Universities for Research in Astronomy, Inc., under cooperative agreement with the National Science Foundation.
} 


\subsection{Initial conditions and assumptions}

We first consider different scenarios in order to try to simulate the morphology of the SNR 3C 400.2. As a first step, models of two interacting SNR were considered, but the final morphology is always elliptical, and does not show two overlapping shells, as observed. As our results are similar to the ones obtained by Ikeuchi (1978) and Jones et al. (1979), we do not show them in the present paper.

We have also simulated a SN explosion close to an interface which separates two media with different densities. Several distances from the SN explosion site to the interface were tested and it was also considered that the SN explosion takes place in the low density medium, right on the interface, or in the high density medium. After several tests and taking into account the observational evidence (e.g. the Giacani et al. 1998 HI study, which shows the existence of an interface between two regions of different densities, going right through the position of the SNR), we find that the more adequate model for describing the morphology of the SNR 3C 400.2 is the following: a single SN event occurring in a dense medium, close to an interface with a lower density region.

In principle, it would be possible to explore models of two interacting explosions occurring in a region with an interface between a high- and a low-density medium. However, we do not explore this possibility as it is more complex, and does not appear to be necessary for modelling the SNR 3C 400.2.

The calculations were carried out with the new yguazú$a$ code, which is described above. Due to the symmetry of the problem, we have integrated the cylindrically symmetric gas dynamic equations. In our simulations, we used a 5 -level binary adaptive grid with a maximum resolution of $9.7 \times 10^{-2} \mathrm{pc}$, in a $50 \times 25 \mathrm{pc}($ axial $\times$ radial $)$ computational domain. We also carried out a $3 \mathrm{D}$ simulation, with a maximum resolution of $6.5 \times 10^{-2} \mathrm{pc}$ in a domain of $8.3 \mathrm{pc}$ along the three coordinate axes. This simulation was made in order to analyze the early stages of the interaction between the SNR shock wave and the interface between the high- and low-density regions, and to show that the resulting flow does not depend on the assumption of axisymmetry.

In our framework, the dense and light media have number densities of $20 \mathrm{~cm}^{-3}$ and $4 \mathrm{~cm}^{-3}$ (following the HI study of Giacani et al. 1998), respectively. The temperature for the low density medium was taken to be $1 \times 10^{4} \mathrm{~K}$ and both media are in pressure equilibrium (giving a temperature of $2000 \mathrm{~K}$ for the high-density medium). The distance from the SN explosion site to the interface was set to 0 and $\pm 1.17 \mathrm{pc}$. We choose an initial radius and explosion energy for the SNR of 1 pc and $1 \times 10^{51}$ erg, respectively. Moreover, we consider that the remnant gas is fully ionized while the gas of the surrounding ISM is neutral.

In order to compare our numerical results with observations, we also generated $\mathrm{H} \alpha$ emission maps taking into consideration the contributions of the recombination

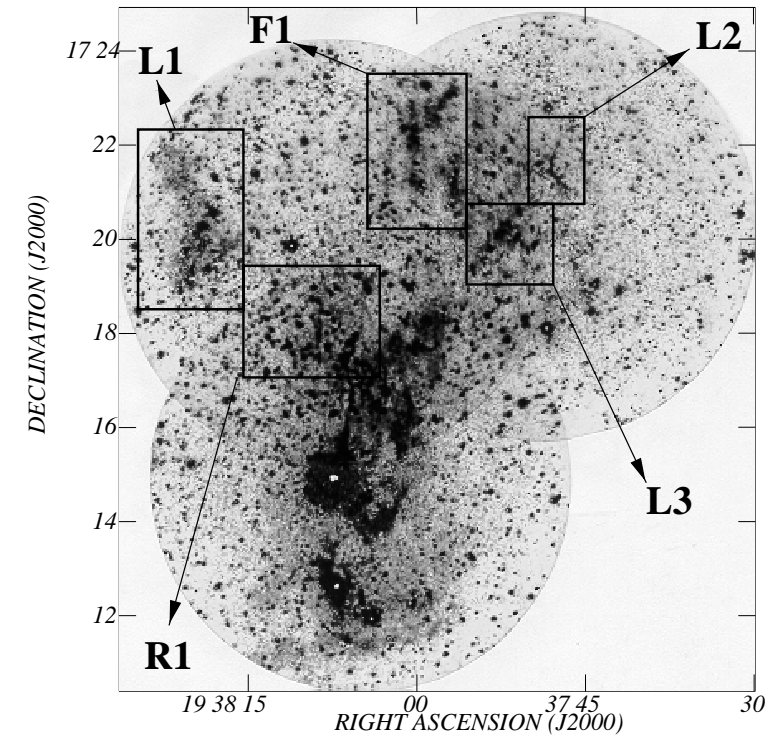

Fig. 1. H $\alpha$ CCD mosaic image covering the north-west region of the SNR 3C 400.2. The locations of the features cited in the text, the region $\mathrm{R} 1$, located at $19^{\mathrm{h}} 38^{\mathrm{m}} 10^{\mathrm{s}} ; 17^{\circ} 18^{\prime}$; the "fork"centered at J2000: $19^{\mathrm{h}} 38^{\mathrm{m}} ; 17^{\circ} 22^{\prime}$ (labeled F1) and the wedge like structures centered approximately at $19^{\mathrm{h}} 38^{\mathrm{m}} 20^{\mathrm{s}} ; 17^{\circ} 20^{\prime}$; $19^{\mathrm{h}} 37^{\mathrm{m}} 47^{\mathrm{s}} ; 17^{\circ} 20^{\prime} 21^{\prime \prime}$ and $19^{\mathrm{h}} 37^{\mathrm{m}} 50^{\mathrm{s}} ; 17^{\circ} 20^{\prime}$ (labeled L1, L2 and L3, respectively), are indicated.

cascade as well as collisional excitations from the ground state of $\mathrm{H}$.

\section{Results}

\section{1. $\mathrm{H \alpha}$ CCD mosaic}

A composite image from the three $\mathrm{H} \alpha$ images was constructed to obtain a deep mosaic image of the NW region of SNR 3C 400.2 that covers the overlapping zone between the two apparent radio shells of this SNR (see Dubner et al. 1994). The resulting mosaic image is shown in Fig. 1, which covers a field of $13^{\prime} .5 \times 14^{\prime} .5$ equivalent to $11 \times 11.8 \mathrm{pc}$ (assuming a distance of $2.8 \mathrm{kpc}$, Giacani et al. 1998). To generate the CCD mosaic, we identified and employed the stars present in our field as references: SAO 105081 (the brightest star at $\left.19^{\mathrm{h}} 38^{\mathrm{m}} 27^{\mathrm{s}} .18 ; 17^{\circ} 15^{\prime} 26^{\prime \prime} .3\right)$, GSC 1606_01128 (at $19^{\mathrm{h}} 38^{\mathrm{m}} 18.04 ; 17^{\circ} 20^{\prime} 49^{\prime \prime} .7$, located in the center of L1 feature, see Fig. 1), GSC 1606_02309 $\left(19^{\mathrm{h}} 37^{\mathrm{m}} 56.77\right.$; $17^{\circ} 23^{\prime} 8^{\prime \prime} .3$, located to the north of F1 feature, Fig. 1), GSC 1606_00830 $\left(19^{\mathrm{h}} 37^{\mathrm{m}} 47^{\mathrm{s}} .21 ; 17^{\circ} 21^{\prime} 10^{\prime \prime} 4\right.$, located to the east of the L2 feature, Fig. 1) and GSC 1606_00462 $\left(19^{\mathrm{h}} 37^{\mathrm{m}} 59 \mathrm{~s} .18,17^{\circ} 17^{\prime} 52^{\prime \prime} \cdot 2\right.$, located in the center of the observed field).

The mosaic image presented here is deeper than previous images reported in the literature (e.g. Winkler et al. 1993). This deeper $\mathrm{H} \alpha$ image allows us to obtain a more significant comparison between the observed morphology of the region where the two shells overlap and our theoretical models. 


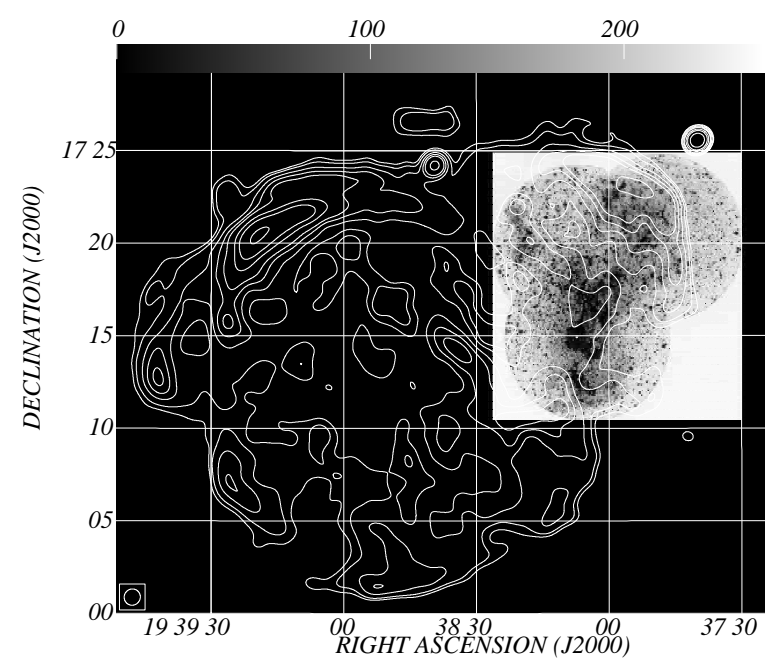

Fig. 2. Overlay of the $\mathrm{H} \alpha$ emission of the north-west region of SNR 3C 400.2 (in gray scale) and a radio continuum map (at $1465 \mathrm{MHz}$, Dubner et al. 1994) with contours of 3, 4, 6, 8, 10, $15,20,30 \mathrm{~mJ} /$ beam.

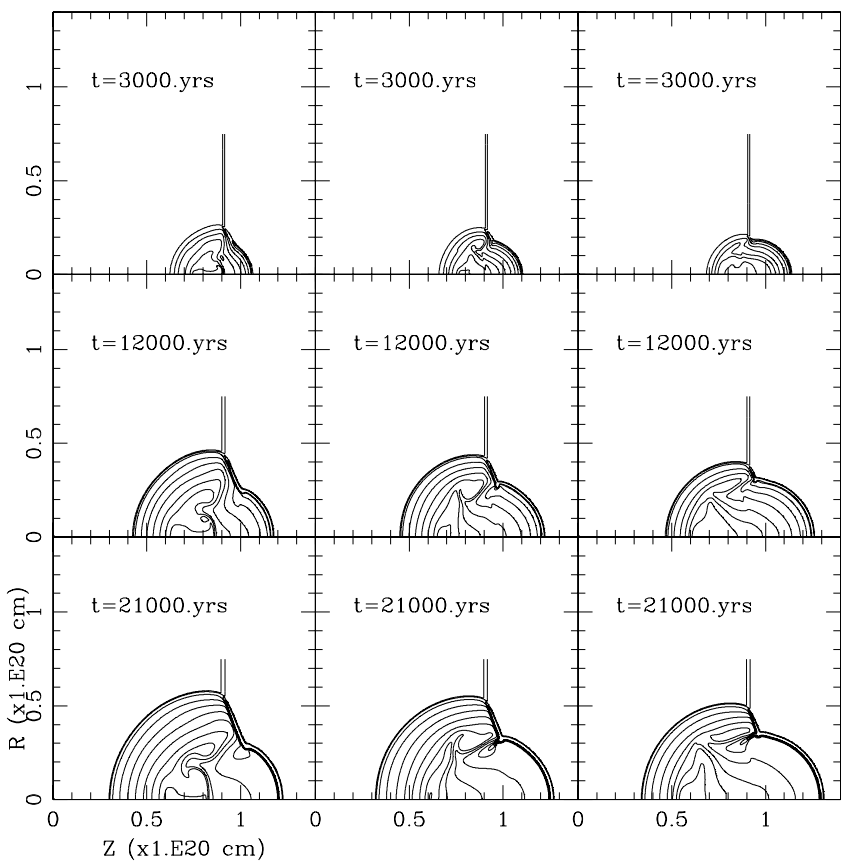

Fig. 3. Comparison between the different cases of a single SN explosion close to an interface. We show the cases in which the explosion took place in the low density medium (the three left panels), right on the interface separating both media (the three middle panels) and in the dense medium (the three right panels). For each model, we present the density stratification for times $t=3000,12000$ and 21000 yr. In each panel, the dense medium is on the right of the vertical line (i.e., the interface between the two media). Two successive contours correspond to a factor of 2 change in the density.

In the observed region, we can confirm the presence of faint features and structures, some of which appear in previous images (Winkler et al. 1993, see Figs. 1, 4 and 5 of their work). The optical emission is composed of bright features and filaments embedded in a faint and diffuse

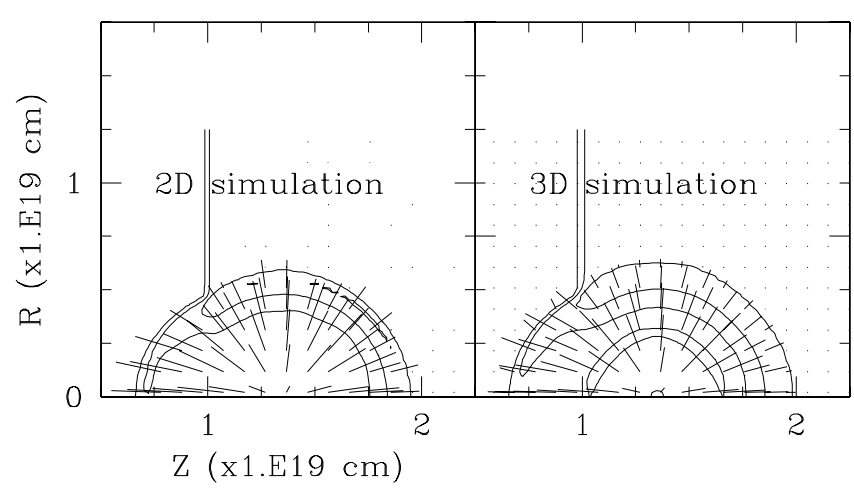

Fig. 4. Comparison of the density stratification and velocity field, at $t=200 \mathrm{yr}$, between the $3 \mathrm{D}$ simulation (right panel), and a $2 \mathrm{D}$ simulation with the same spatial resolution (left panel).

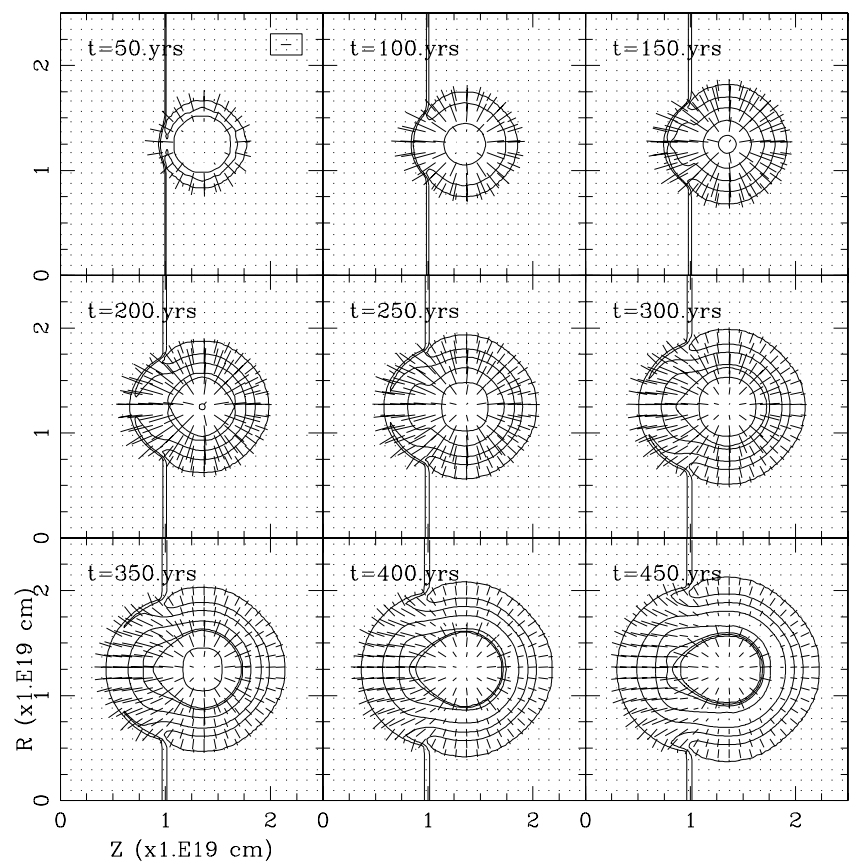

Fig. 5. Density contours and velocity field of the evolution of a SNR expanding into an environment with a one step density profile obtained from the 3D numerical simulation. The vertical line is the interface between the high- (right hand side) and the low-density media (left hand side). The length of the horizontal line in the box (in the top right hand corner of the first frame) corresponds to a velocity of $2000 \mathrm{~km} \mathrm{~s}^{-1}$. At $t=200$ and $250 \mathrm{yr}$, a partial collimation is produced.

emission (for example, see region R1 in Fig. 1). These filaments have "arc" or "wedge" shapes, as can be seen in the northern sector (farther than $\delta=17^{\circ} 16^{\prime}$ ), particularly in the structure centered at $19^{\mathrm{h}} 38^{\mathrm{m}} ; 17^{\circ} 22^{\prime}$ (structure F1) which is formed by several filaments giving the appearance of a "fork" and the features centered approximately at $19^{\mathrm{h}} 38^{\mathrm{m}} 20^{\mathrm{s}} ; 17^{\circ} 20^{\prime} ; 19^{\mathrm{h}} 37^{\mathrm{m}} 47^{\mathrm{s}} ; 17^{\circ} 20^{\prime} 21^{\prime \prime}$ and $19^{\mathrm{h}} 37^{\mathrm{m}} 50^{\mathrm{s}}$; $17^{\circ} 20^{\prime}$ (the L1, L2 and L3 features), respectively, which are "wedge" shaped (see Fig. 1). These filaments can be produced by collisional excitation right behind radiative shocks or by recombination processes in the cooling region. 
(a)

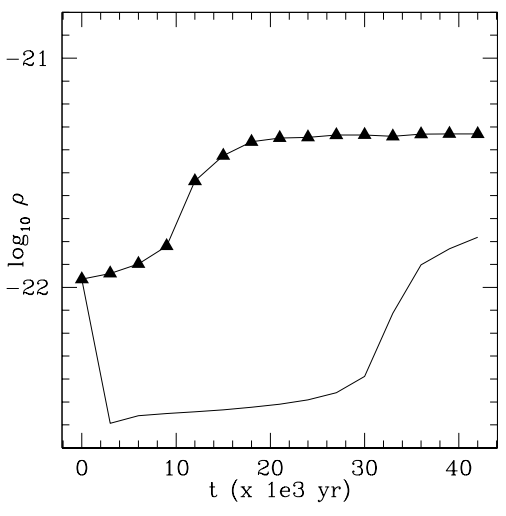

(b)

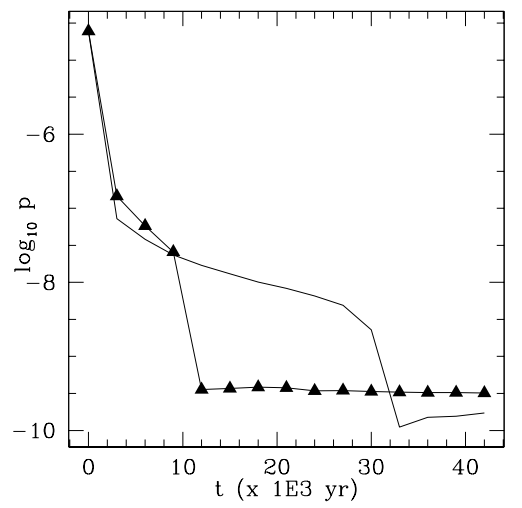

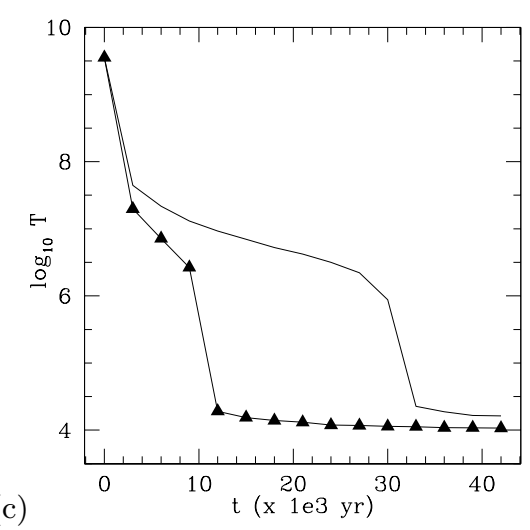

(c)

Fig. 6. Comparison of the time evolution versus the logarithm of the density, pressure and temperature (panels a), b) and c), respectively) right behind the shock waves of the small (represented with triangles and solid lines) and the large shells (represented with solid lines).

In Fig. 2 we show an overlay of our $\mathrm{H} \alpha$ image with some contours of the radio image of the SNR 3C 400.2, at $1465 \mathrm{MHz}$ (Dubner et al. 1994). Here, we can observe how the extended and diffuse emission follows the curvature of the large shell, and is also observed right behind the shock wave of the small shell.

The detection of optical filaments in this zone is also useful because it will allow us to have kinematical information on these filaments. A preliminary analysis of the data obtained with the PUMA equipment in its interferometric mode reveals that the small shell has an expansion velocity which is smaller than that of the large shell $\left(\sim 40 \mathrm{~km} \mathrm{~s}^{-1}\right.$ vs. $\left.60 \mathrm{~km} \mathrm{~s}^{-1}\right)$, the latter having been determined by Rosado (1983).

\subsection{Numerical simulations}

Considering the initial conditions described above (Sect. 3) we carried out numerical simulations, with different spatial resolutions, in order to study a SNR generated by an explosion which took place close to an interface between a high- and a low-density media.

Some preliminary results from these simulations are presented in Fig. 1 of de la Fuente et al. (2001).

Figure 3 shows a comparison between simulations carried out considering that the $\mathrm{SN}$ explosion took place in the light medium (left column), right on the interface (middle column) and in the dense medium (right column). In the calculations, the lower density medium always lies to the left of the interface between the two media. We see that the "left" shell has a larger radius when the SN explosion takes place in the less dense medium. However, the appearance of two overlapping shells is better achieved in the two other cases.

Figure 5 shows the density stratifications and velocity fields from the $3 \mathrm{D}$ simulation. In this figure, we see that at early times $(t=50 \mathrm{yr})$, the left part of the SNR shock wave catches up with the interface between the dense and light media. Then, this part of the SNR shock front increases its velocity, producing a break out in the remnant surface, generating a bubble. The remnant starts to have the appearance of two partially overlapped shells.

An interesting phenomenon is observed close to the symmetry axis. At $t=200$ and $250 \mathrm{yr}$ frames (see Fig. 5) one observes a collimation of the internal gas (which has a high temperature), which looks like a "nozzle". This hot gas starts to fill up the bubble and its velocity increases to values of $1 \times 10^{4} \mathrm{~km} \mathrm{~s}^{-1}$. The velocity of the collimated gas remains larger than the shock wave velocity of the large shell until approximately 20000 yr. However, the Mach number of the "nozzle" gas is smaller than 1, and this collimated gas is not able to generate a "jet" (in agreement with the work of Arthur \& Falle 1991).

There is little doubt that this axial collimation of the flow is real, as this result is obtained from a full 3D simulation, in which there are no possible artifacts due to an assumption of axisymmetry for the flow. Actually, we find that a very similar flow is obtained from an axisymmetric simulation, as can be seen from Fig. 4, which shows a comparison between the density stratifications and flow fields obtained for $t=200 \mathrm{yr}$ from the 3D simulation and a $2 \mathrm{D}$ (axisymmetric) simulation with the same spatial resolution.

The two apparent shells have very different evolutions. On the one hand, they sweep up gas with different densities, which implies that the part of the SNR expanding into a dense medium will have a rapid evolution (hereafter "the small shell"), according to the standard SNR evolution scheme (Woltjer 1970, 1972). On the other hand, the collimation of the hot gas produces an extra injection of energy into the left "shell" (hereafter "the large shell"), giving the appearance of having a SNR with a higher initial explosion energy. However, at early times ( $t=200 \mathrm{yr})$, the evolution of the large shell cannot be described by the standard SNR evolution, because this shell is driven by the gas of the "nozzle".

Figure 6 shows the density, pressure and temperature [panels a), b), and c), respectively] right behind the SNR shock waves propagating into the low- and the high-density media. Interestingly, the behaviour of these 


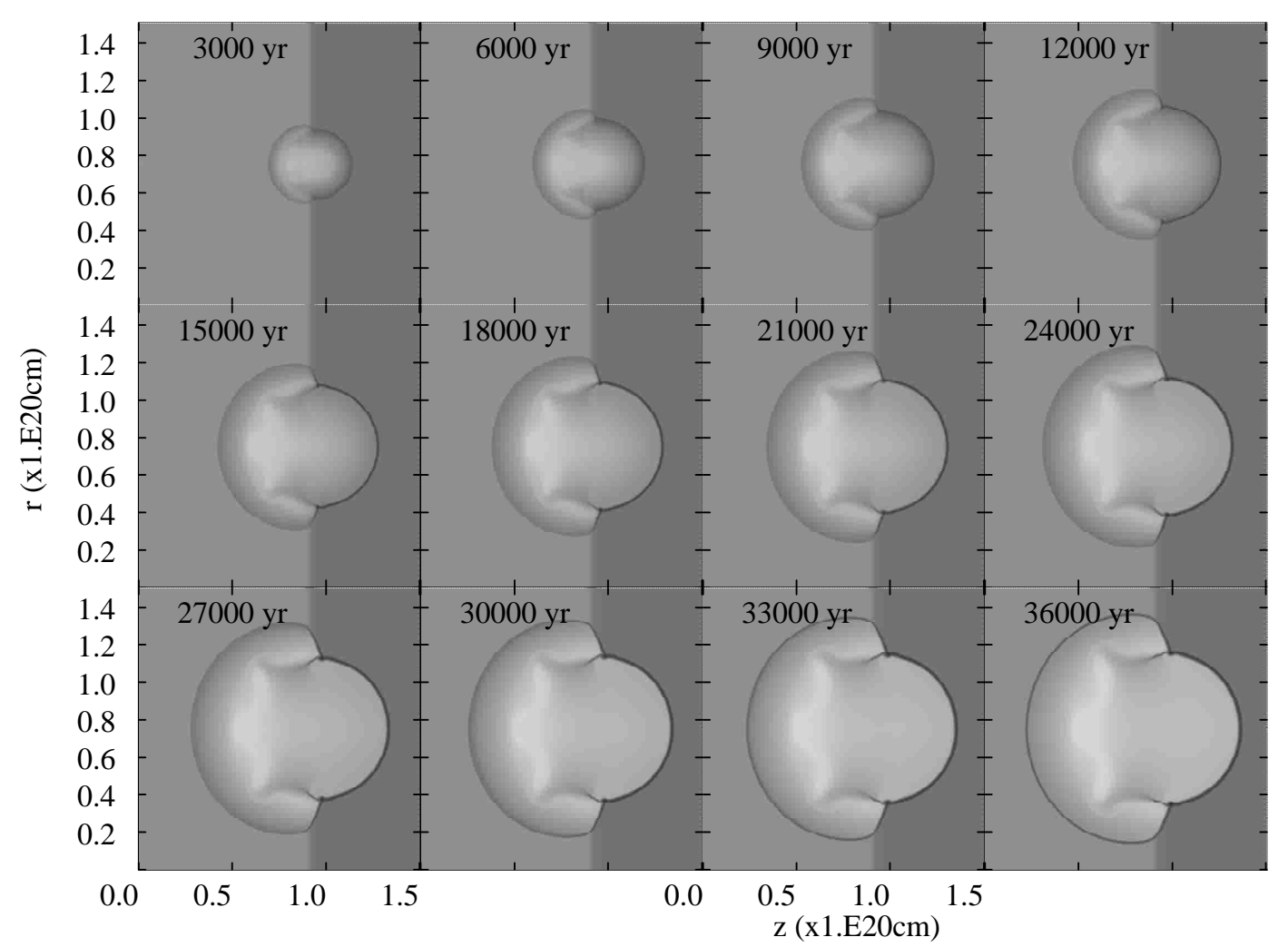

Fig. 7. Evolution of the density stratification of a SNR expanding into an environment with an one-step density profile. The gray-scale corresponds to a range from $1 \times 10^{-2} \mathrm{~cm}^{-3}$ (white) to $1 \times 10^{4} \mathrm{~cm}^{-3}$ (black). The scale is logarithmic.

density, pressure and temperature profiles seems to be independent of where the SN explosion took place.

Figure $6 \mathrm{~b}$ shows that at early times there is pressure equilibrium between the regions behind the shock waves of the two "shells". However, at $t \simeq 12000 \mathrm{yr}$, this equilibrium is broken because the small shell enters the radiative phase of SNR evolution and the pressure behind the shock front decreases by 2 orders of magnitude.

The shock wave of the small shell starts to become radiative at $t \simeq 12000 \mathrm{yr}$ (notice that the pressure and temperature rapidly decrease in Figs. 4b and c, respectively). This time is in agreement with the analytical estimate (approximately $10700 \mathrm{yr}$ ) for the beginning of the radiative phase of SNR evolution (for an initial explosion energy $E_{0}=1 \times 10^{51} \mathrm{erg}$ and an ISM density of $20 \mathrm{~cm}^{-3}$ ), which is approximately given by:

$\tau_{\text {rad }}=\left[\frac{4.56 \times 10^{7}}{\left(1+x_{H}\right) T_{4}}\left(\frac{E_{51}}{n_{0}}\right)^{2 / 5}\right]^{5 / 6} \mathrm{yr}$,

where $T_{4}$ is the gas temperature in units of $10^{4} \mathrm{~K}, E_{51}$ is the initial SN explosion energy in units of $10^{51} \mathrm{erg}$, $x_{\mathrm{H}}$ is the ionization fraction and $n_{0}$ is the unperturbed ISM density. Equation (1) is obtained setting the temperature just behind the SNR shock wave to $10^{6} \mathrm{~K}$ (see Rohlfs \& Wilson 1996).

The density just behind the shock wave of the small shell increases by a factor of 15 , at $t \simeq 15000 \mathrm{yr}$, due to the onset of the radiative phase. Numerically, this shell is resolved by $\sim 5$ grid points, so that the numerical value of the compression should be correct, at least in order of magnitude. The large shell becomes radiative later, at $t \simeq$ $35000 \mathrm{yr}$. This value is larger than the one given by Eq. (1) $\left(t \simeq 18500 \mathrm{yr}\right.$ for a $\left.n_{0}=4 \mathrm{~cm}^{-3}\right)$. This behaviour is due to the extra energy injection produced by the collimation of the hot gas, which rejuvenates the large shell.

Figure 7 shows the density time evolution obtained for larger times from the low resolution simulation. Part of the gas which has entered the large shell, begins to push out the outer part of the interface between the light and dense media. This flow moves in the opposite direction to the "nozzle" gas (see Fig. 7), generating zones with high velocity gradients. This part of the interface between both media becomes radiative practically at the same time as the shock front of the small shell.

Two dense filaments appear at $t \simeq 18000 \mathrm{yr}$ (Fig. 7). In the following frames we can see that these filaments increase their lengths inwards, towards the center of the large shell. The filaments are found in a region with high velocity shear, and they begin to exhibit wavy structures, probably produced by Kelvin-Helmholtz instabilities.

Maps of the normalized $\mathrm{H} \alpha$ emission at several times are displayed in Fig. 8. These maps were obtained by integrating the $\mathrm{H} \alpha$ emissivity along lines of sight. Before $t \simeq 12000 \mathrm{yr}$, no $\mathrm{H} \alpha$ emission was detected. The fact that the observed $\mathrm{H} \alpha$ images of $3 \mathrm{C} 400.2$ present arc like diffuse emission, appears to indicate that the symmetry axis (joining the centers of the two apparent shells) is inclined at an angle $\phi$ respect to the plane of the sky. We have 


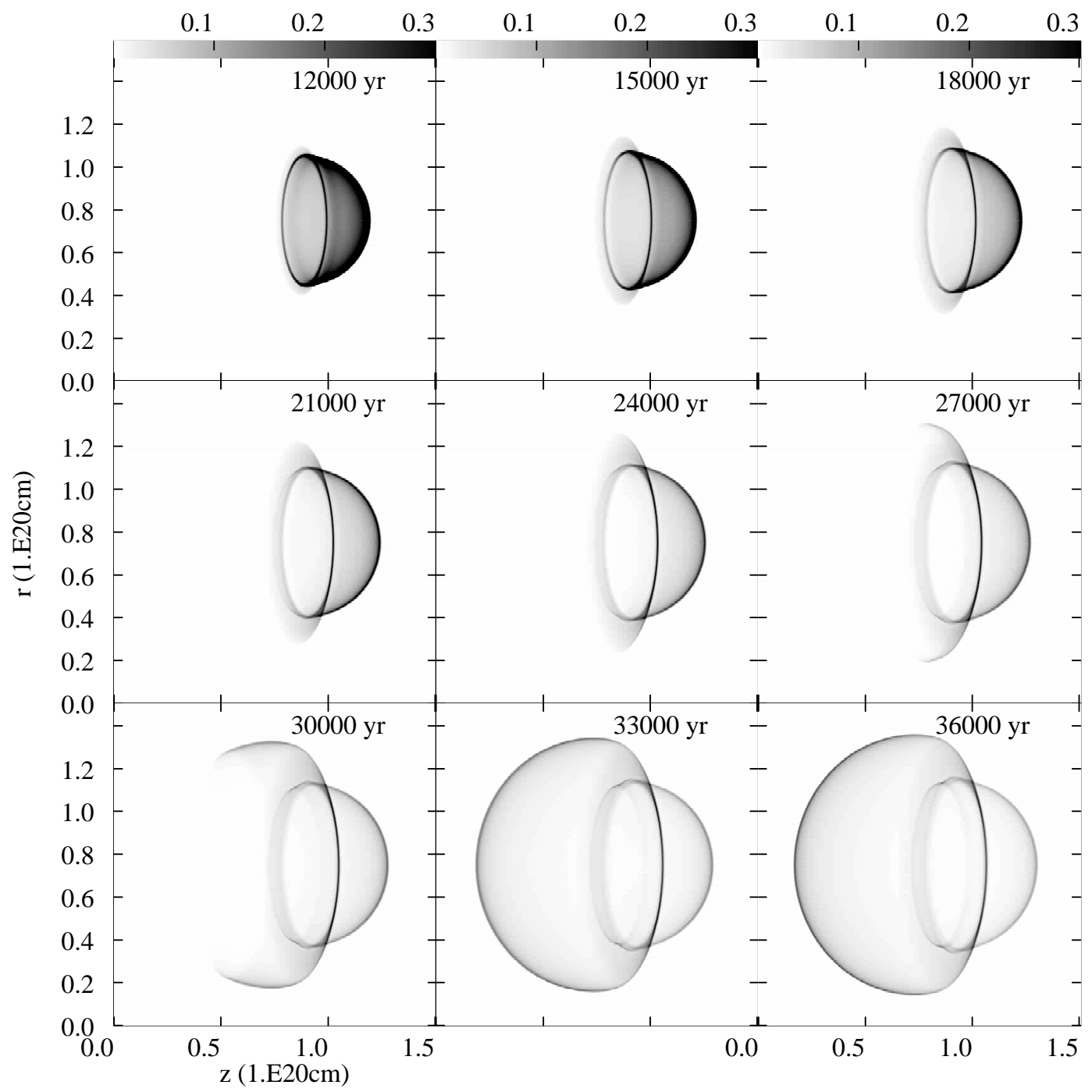

Fig. 8. Maps of the normalized $\mathrm{H} \alpha$ emission. The grey scale is linear and covers the $[0.01,0.3]$ normalized unit range. These maps were obtained for a $\phi=20^{\circ}$ angle between the symmetry axis and the plane of sky.

chosen $\phi=20^{\circ}$ in order to generate the simulated optical emission maps.

$\mathrm{H} \alpha$ emission can be observed after the shock wave of the small shell enters the radiative phase of SNR evolution. Strong emission is observed right behind the shock front of the small shell at $t=12000,15000$ and 18000 yr. Later, the part of the interface between both media, which is pushed out by the returning flow of the "nozzle", begins to exhibit diffuse $\mathrm{H} \alpha$ emission (see the $t=21000,24000,27000$ and $30000 \mathrm{yr}$ frames, Fig. 8) looking like complete and partial rings. Finally, the shock wave of the large shell becomes radiative, emitting in $\mathrm{H} \alpha$ throughout its surface. Comparing the simulated $\mathrm{H} \alpha$ maps with our observations and with the images of Winkler et al. (1993), we conclude that SNR 3C 400.2 seems to have an age of $(26000 \pm 5000)$ yr, because no optical emission was detected behind the shock wave of the large shell.

Due to the fact that the distance to this remnant is not well known (by means of different methods, values for the distance to SNR 3C 400.2 are derived in the range from 2.3 to $6.7 \mathrm{kpc}$ ), a direct comparison between the observed and predicted sizes of the remnant is not particularly useful. However, in this problem there are two characteristic lengths: the radii of the two apparent shells. We can then compare the observed and predicted ratios between these radii. It is remarkable that the ratio between the radii of the large and the small shells of the simulated density maps (Fig. 7 ) is $\simeq 1.5$ (at $t \simeq 24000 \mathrm{yr}$, the time at which the best agreement between the observed and the simulated $\mathrm{H} \alpha$ maps is obtained), agrees very well with the observed ratio of $\simeq 1.58$.

\section{Conclusions}

We have carried out 2D and 3D numerical simulations in order to explain SNR 3C 400.2 morphology in radio continuum. Based on observational evidence (e.g. the analysis of the HI distribution around this remnant of Giacani et al. 1998) and after several tests, we find that a scenario in which a $\mathrm{SN}$ explosion took place in a dense medium and 
close to an interface which separates this dense medium from a less dense one, is adequate for describing this remnant.

These simulations were done employing the new yguazú- $a$ code (Raga et al. 2000). Due to the fact that in the case of SNR 3C400.2 the synchrotron emission seems to be tracing the position of the shock waves (as it shows a structure of well defined shells), we can compare the radio continuum images of 3C 400.2 with the shock structures predicted from the numerical simulations. We do this by comparing the radio continuum images with the predicted density stratifications, which also show the morphologies of the shock waves very clearly. From this comparison, we conclude that an "explosion in an interface" scenario does reproduce the radio structure of 3C 400.2 satisfactorily. A more realistic comparison between the numerical model and the observations should involve obtaining a prediction of the spatial distribution of the synchrotron emission, but we find that this is not yet possible as we do not have enough knowledge of the spatial distribution of the magnetic field within the remnant and in the surrounding environment.

We also obtained a $\mathrm{H} \alpha \mathrm{CCD}$ mosaic of the NW region of the SNR 3C 400.2 in which bright and diffuse optical filaments are observed (Fig. 1). Part of this emission was not well detected in previously published images. Some of the diffuse filaments are located right behind the shock wave of the small shell. Other filaments seem to continue the circumference of the large shell, in the region of overlap between the two apparent shells. Bright, well defined filaments are seen superimposed on this diffuse emission.

Furthermore, we simulate the $\mathrm{H} \alpha$ emission, to compare the model directly with the observed optical images. We obtain normalized $\mathrm{H} \alpha$ emission maps which are in qualitative agreement with the observations. The best agreement is obtained assuming that the symmetry axis of the remnant is at a $\phi=20^{\circ}$ angle with respect to the plane of sky (Fig. 8). Filaments looking like arcs appear right behind the shock wave of the small shell in qualitative agreement with the observed $\mathrm{H} \alpha$ image. This behaviour is observed in the simulated maps for time integrations $t=12$ 000, 15 000, 18000, 21000 and $24000 \mathrm{yr}$ (see Fig. 8). From the simulated $\mathrm{H} \alpha$ maps, diffuse and strong emission is observed from the part of the "interface" between both media, which becomes radiative after being pushed out by the gas of the large shell. This feature is observed in the images obtained by Winkler et al. (1993), and is only partially covered in our CCD mosaic. No optical emission was detected from the material right behind the shock wave of the large shell (see the images of Winkler et al. 1993). In view of these results, from a comparison with our simulations this remnant seems to be younger than previously estimated (e.g. $1 \times 10^{5}$ yr, Rosado 1983), and we obtain an estimate for the age of the SNR 3C 400.2 of 26000 yr. Approximately at this time, the observed ratio between the diameters of both shells is well reproduced by the simulation (1.58 obtained from the radio observations against 1.5 from our simulations).
Figure 1 shows small $\mathrm{H} \alpha$ features, which probably are the results of the presence of inhomogeneities in the surrounding ISM, or are the results of some kind of hydrodynamic instability. These small scale structures do not appear in our simulation.

An interesting result is that the small shell seems to follow the standard SNR evolution in a dense medium, but that this is not the case for the large shell. The latter shell evolved in a different way due to the collimation of the hot gas, passing from the small apparent shell to the large one. This gas has a "nozzle" type flow, which pushes out the material of the large shell as is shown by our 3D simulations. This result is in agreement with the work by Arthur \& Falle (1991).

It is in principle also possible to carry out comparisons between the observed X-ray images of 3C 400.2 (Matsui \& Long 1985; Seward 1990) and the corresponding predictions obtained from the numerical simulations. We have attempted to carry out such a comparison, but we do not obtain satisfactory results. While the X-ray maps of this object show a centrally peaked intensity distribution, the numerical simulations predict a shell-like X-ray morphology. This discrepancy between the observations and the theoretical prediction might be due to the effects of thermal conduction (not included in our simulations) which might have a strong effect on the temperature and density stratification of the hot bubbles.

As future steps we will complete the kinematical analysis of the PUMA data, and we will include the process of thermal conduction in our code, in order to simulate the X-ray emission. This mechanism was invoked by Shelton et al. (1999); Cox et al. (1999), to explain the central X-ray emission of the SNR W44 (another example of a SNR with central X-ray emission and radio shell morphology).

Acknowledgements. We thank Gloria Dubner for providing us with her $1465 \mathrm{MHz}$ image of 3C 400.2 and Liliana Hernández for computer help. We also acknowledge the referee, Dr. V. Dwarkadas for several helpful suggestions. PV and AR acknowledge support from the CONACyT grant 32753-E. AR acknowledges support from a fellowship of the John Simon Guggenheim Memorial Foundation. E. de la F. wishes to acknowledge financial support from CONACyT grant 124449 and from DGEP-UNAM. This work has also been financed by grant IN104696 from DGAPA(UNAM).

\section{References}

Arthur, S. J., \& Falle, A. E. G. 1991, MNRAS, 251, 93

Blair, W. P., \& Long, K. S. 1988, PASP, 100, 461

Condon, J. J., Broderick, J. J., \& Seielstad, G. A. 1989, AJ, 97, 1064

Cox, D. P, Shelton, R. L., Maciejewski, W., et al. 1999, ApJ, 524,179

de la Fuente, E., Velázquez, P. F., Rosado, M. \& Raga, A. C. 2001, in Ionized Gaseous Nebulae, RMxAC, in press.

Dickel, J. R., \& De Noyer, L. K. 1975 AJ, 80, 437 
Dubner, G. M., Giacani, E. B., Goss, W. M., \& Winkler, P. F. Raga, A. C., Cabrit, S., Dougados, C., \& Lavalley, C., 2001, 1994, AJ, 108, 207 A\&A, 367, 959

Dubner, G. M., Holdaway, M., Goss, W. M., \& Mirabel, I. F. Rohlfs, K \& Wilson, T. L. 1996, in Tools of Radio Astronomy 1998, AJ, 116, 1842

(Springer, Germany), 267

Giacani, E. B., Dubner, G., Cappa, C., \& Testori, J. 1998, A\&AS, 133, 61

Goss W. M., Siddesh, S. G., \& Swchartz, U. J. 1975, A\&A, 43, 459

Holden, D. J., \& Caswell, J. L. 1969, MNRAS, 143, 407

Ikeuchi, S. 1978, PASJ, 30, 563

Jones, E. M., Smith, B. W., Straka, W. C., Kodis, J. W., \& Guitar, H. 1979, ApJ, 232, 129

Le Coarer, E, Rosado, M. Georgelin, Y., Viale, A., \& Goldes, G. 1993, A\&A 280, 365

Long, K. S. \& Blair, W. P., White, R. L., \& Matsui, Y. 1991, ApJ, 373, 567

Matsui, Y. \& Long, K. S. 1985, in The Crab Nebula and Related Supernova Remnants, ed. M. C. Kafatos, \& R. B. C. Henry (Cambridge University Press, Cambridge), 211

Milne, D. K. 1970, Australian J. Phys., 23, 245

Raga, A. C., Mellema, G., Arthur, S. J., et al. 1999, RMxAA, 35,123

Rosado, M. 1983 RMxAA, 8, 59

Rosado, M., Langarica, R., Bernal, A., et al. 1995, RMxAC, 3, 263.

Sabbadin, F., \& D'Odorico, S. 1976, A\&A, 49, 119

Seward, F. D. 1990, ApJS, 73, 781.

Shelton, R. L., Cox, D. P, Maciejewski, W., et al. 1999, ApJ, 524, 192

Tenorio-Tagle, G, Bodenhaimer, P., \& Yorke, H. W. 1985, A\&A, 145, 70

van den Bergh, S., Marscher, A. P., \& Terzian, Y. 1973, ApJSS, 227,26

Van Leer, B. 1982, ICASE report No. 82-30

Velázquez, P. F., \& Raga, A. C. 2000, A\&A, 362, 780

White, R. L., \& Long, K. S. 1991, ApJ, 373, 567

Willis, A. G., 1973, A\&A, 26, 237

Winkler F. P., Olinger, M. T., \& Westerbeke, S. A. 1993, ApJ, 405, 608

Woltjer, L. 1970, IAU Symp., 39, 229

Woltjer, L. 1972, ARA\&A, 10, 129

Raga, A. C., Navarro-González, R., \& Villagrán-Muniz, M. 2000, RMxAA, 36, 67 